\title{
Predictive Factors of Response and Overall Survival in Patients with Castration-Resistant Metastatic Prostate Cancer Undergoing ${ }^{177}$ Lu-PSMA Therapy
}

\author{
Hojjat Ahmadzadehfar and Markus Essler \\ Department of Nuclear Medicine, University Hospital Bonn, Bonn, Germany
}

$\mathbf{P}$ rostate-specific membrane antigen (PSMA) is highly expressed in prostate epithelial cells and strongly upregulated in prostate cancer. Therefore, PSMA is an appropriate target for the diagnosis and therapy of prostate cancer and its metastases. Since 2013, an increasing number of centers worldwide have begun using radioligand therapy (RLT) with ${ }^{177}$ Lu-PSMA (1-4). Several studies have demonstrated the safety and low toxicity profile of ${ }^{177} \mathrm{Lu}-$ PSMA for the therapy of patients with metastatic castration-resistant prostate cancer $(1,3,5-8)$. Up to $80 \%$ of these patients will respond to treatment with ${ }^{177} \mathrm{Lu}-\mathrm{PSMA}$ as measured by any decline in prostate-specific antigen (PSA) $(1,5,6,8-13)$. Studies involving ${ }^{177} \mathrm{Lu}-\mathrm{PSMA}-617$ and ${ }^{177} \mathrm{Lu}-\mathrm{PSMA}-\mathrm{I} \& \mathrm{~T}$ have observed a reduction in PSA levels of $50 \%$ or more in $32 \%-60 \%$ of patients.

Various pretherapeutic parameters have been evaluated to determine whether there are predictors of response to RLT. Ferdinandus et al. (10) evaluated the prognostic value of some pretherapeutic parameters on therapy response based on changes in PSA after the first cycle of RLT. Their multivariate analysis of these parameters, in which any decrease in PSA after 2 mo was considered to represent a response, showed that patients with a high platelet count or a regular need for analgesics had a significantly lower response rate to the first cycle of RLT. According to Ferdinandus et al., the $\mathrm{SUV}_{\max }$ of ${ }^{68} \mathrm{Ga}$-PSMA-11 was not a significant predictor of the response to ${ }^{177} \mathrm{Lu}-\mathrm{PSMA}$ RLT. One explanation could be that although more aggressive tumors may express higher PSMA uptake, this uptake level does not correlate with the response rate because of the rapid progression of the disease. Other explanations could be differences in the residency time of radioactivity in the metastases and differences in the compounds used for imaging and treatment.

It seems that prior therapies have no significant impact on response rate. According to the multicenter German Study, relevant independent predictors of biochemical response included an alkaline phosphatase level of less than $220 \mathrm{U} / \mathrm{L}$, the absence of visceral metastases, and a higher number of therapy cycles (3). About $88 \%-93 \%$ of patients with a PSA response after the first cycle responded to further cycles $(6,9,12)$, whereas approximately $40 \%$ of nonresponders responded to further therapy cycles $(9,12)$.

Received Mar. 9, 2018; revision accepted Apr. 9, 2018.

For correspondence or reprints contact: Hojjat Ahmadzadehfar, Department of Nuclear Medicine, University Hospital Bonn, Sigmund-Freud-Strasse 25, 53127 Bonn, Germany.

E-mail: hojjat.ahmadzadehfar@ukbonn.de

Published online Apr. 13, 2018.

COPYRIGHT (c) 2018 by the Society of Nuclear Medicine and Molecular Imaging. DOI: 10.2967/jnumed.118.209270
Response to RLT based on PSA changes is actually one of the most significant parameters of overall survival, according to multiple studies showing that the median overall duration of survival was significantly longer for patients who exhibited a PSA decline 2 mo after the first cycle than for patients without a PSA decline (median, 63-71 wk vs. 33-47 wk, respectively) $(9,13,14)$. According to various guidelines and trials, response to a therapy is defined as a decline in PSA level by at least 50\%; however, in patients being treated with ${ }^{177} \mathrm{Lu}-\mathrm{PSMA}$ as a last option, the disease is typically in a very aggressive phase with a rapidly rising PSA level before RLT. Thus, a PSA level that is unchanged 2 mo after the first cycle of RLT could be considered a response to treatment. In one of our recently published studies (13), we reported the predictive factors of overall survival in 104 patients who had received all the approved therapies. In that study, in addition to a PSA decline after the first cycle, an alkaline phosphatase level with a cutoff of $220 \mathrm{U} / \mathrm{L}$ and a cumulative administered activity with a cutoff of $18.8 \mathrm{GBq}$ were further significant prognostic factors of overall survival.

Thus far, a standard administered activity of ${ }^{177}$ Lu-PSMA has not been determined by prospective trials. A wide range of activities - up to $9.3 \mathrm{GBq}$ - has been used in safety and toxicity trials reported in the literature, and it seems that there is no correlation between initial PSA response and the amount of therapeutic activity $(3,15)$. The fact that some studies have found a negative correlation between the amount of tumor involvement and the dose absorbed by nontarget organs encourages us to increase the activity for at least the first cycle in patients with high tumor involvement $(16,17)$. This issue should continue to be investigated and proved in prospective studies. It is still unclear why about $20 \%$ of patients do not respond to ${ }^{177} \mathrm{Lu}$-PSMA therapy; however, there is no treatment option with a $100 \%$ response rate. One reason could be that these patients have more aggressive types of prostate cancer with neuroendocrine differentiation or with somatic genomic alterations or specific germline mutations, such as BRCA2 (18).

The specific amount of ${ }^{177}$ Lu-PSMA activity may also be an important issue in determining therapy response. ${ }^{177} \mathrm{Lu}-\mathrm{PSMA}$ therapy is not curative; therefore, the combination of RLT with other synergistic therapies, such as immunotherapy, may increase its efficacy (19). At the moment, according to the published retrospective studies, the following criteria may be the most suitable for patient selection: histologically proven prostate carcinoma; nonresectable metastases; tumor progression under treatment according to current guidelines; PSMA expression by the 
metastases, detected by PSMA imaging; sufficient hematologic function (leukocyte count $>2.0 \times 10^{9} / \mathrm{L}$, thrombocyte count $>$ $75 \times 10^{9} / \mathrm{L}$ ); normal or slightly decreased renal function (creatinine level $<2$ times the upper standard limit) without any evidence of obstructive renal disease, evaluated by ${ }^{99 \mathrm{~m}} \mathrm{Tc}$-mercaptoacetyltriglycine or ${ }^{99} \mathrm{~m}$ Tc-diethylenetriaminepentaacetic acid; sufficient liver function (aspartate aminotransferase or alanine aminotransferase $<5$ times the upper standard limit); a 6-wk interval after myelosuppressive therapy; and an Eastern Cooperative Oncology Group performance status of 2 or less.

All of above-mentioned data are based on retrospective analysis and therefore should be regarded accordingly and used carefully. A prospective phase 3 study that is being planned by Endocyte (VISION trial) will hopefully augment our knowledge of this therapy.

\section{DISCLOSURE}

No potential conflict of interest relevant to this article was reported.

\section{REFERENCES}

1. Ahmadzadehfar H, Rahbar K, Kurpig S, et al. Early side effects and first results of radioligand therapy with ${ }^{177} \mathrm{Lu}$-DKFZ-617 PSMA of castrate-resistant metastatic prostate cancer: a two-centre study. EJNMMI Res. 2015;5:114.

2. Kabasakal L, AbuQbeitah M, Aygun A, et al. Pre-therapeutic dosimetry of normal organs and tissues of ${ }^{177}$ Lu-PSMA-617 prostate-specific membrane antigen (PSMA) inhibitor in patients with castration-resistant prostate cancer. Eur J Nucl Med Mol Imaging. 2015;42:1976-1983.

3. Rahbar K, Ahmadzadehfar H, Kratochwil C, et al. German multicenter study investigating ${ }^{177} \mathrm{Lu}$-PSMA-617 radioligand therapy in advanced prostate cancer patients. J Nucl Med. 2017;58:85-90.

4. Ahmadzadehfar H, Aryana K, Pirayesh E, et al. The Iranian Society of Nuclear Medicine practical guideline on radioligand therapy in metastatic castrationresistant prostate cancer using ${ }^{177}$ Lu-PSMA. Iran J Nucl Med. 2018;26:2-8.

5. Ahmadzadehfar H, Eppard E, Kurpig S, et al. Therapeutic response and side effects of repeated radioligand therapy with ${ }^{177}$ Lu-PSMA-DKFZ-617 of castrateresistant metastatic prostate cancer. Oncotarget. 2016;7:12477-12488.
6. Kratochwil C, Giesel FL, Stefanova M, et al. PSMA-targeted radionuclide therapy of metastatic castration-resistant prostate cancer with ${ }^{177} \mathrm{Lu}$-labeled PSMA617. J Nucl Med. 2016;57:1170-1176.

7. Yordanova A, Becker A, Eppard E, et al. The impact of repeated cycles of radioligand therapy using $\left[{ }^{177} \mathrm{Lu}\right] \mathrm{Lu}-\mathrm{PSMA}-617$ on renal function in patients with hormone refractory metastatic prostate cancer. Eur J Nucl Med Mol Imaging. 2017;44:1473-1479.

8. Ahmadzadehfar H, Zimbelmann S, Yordanova A, et al. Radioligand therapy of metastatic prostate cancer using ${ }^{177} \mathrm{Lu}$-PSMA-617 after radiation exposure to ${ }^{223}$ Ra-dichloride. Oncotarget. 2017;8:55567-55574.

9. Ahmadzadehfar H, Wegen S, Yordanova A, et al. Overall survival and response pattern of castration-resistant metastatic prostate cancer to multiple cycles of radioligand therapy using $\left[{ }^{177} \mathrm{Lu}\right] \mathrm{Lu}-\mathrm{PSMA}-617$. Eur J Nucl Med Mol Imaging. 2017;44:1448-1454.

10. Ferdinandus J, Eppard E, Gaertner FC, et al. Predictors of response to radioligand therapy of metastatic castrate-resistant prostate cancer with ${ }^{177} \mathrm{Lu}-\mathrm{PSMA}$ 617. J Nucl Med. 2017;58:312-319.

11. Fendler WP, Reinhardt S, Ilhan H, et al. Preliminary experience with dosimetry, response and patient reported outcome after ${ }^{177} \mathrm{Lu}$-PSMA-617 therapy for metastatic castration-resistant prostate cancer. Oncotarget. 2017;8:3581-3590.

12. Rahbar K, Bogeman M, Yordanova A, et al. Delayed response after repeated ${ }^{177}$ Lu-PSMA-617 radioligand therapy in patients with metastatic castration resistant prostate cancer. Eur J Nucl Med Mol Imaging. 2018;45:243-246.

13. Rahbar K, Boegemann M, Yordanova A, et al. PSMA targeted radioligand therapy in metastatic castration resistant prostate cancer after chemotherapy, abiraterone and/or enzalutamide: a retrospective analysis of overall survival. Eur J Nucl Med Mol Imaging. 2018;45:12-19.

14. Ahmadzadehfar H, Schlolaut S, Fimmers R, et al. Predictors of overall survival in metastatic castration-resistant prostate cancer patients receiving $\left[{ }^{177} \mathrm{Lu}\right] \mathrm{Lu}-$ PSMA-617 radioligand therapy. Oncotarget. 2017;8:103108-103116.

15. Rathke H, Giesel FL, Flechsig P, et al. Repeated ${ }^{177}$ Lu-labeled PSMA-617 radioligand therapy using treatment activities of up to $9.3 \mathrm{GBq} . J$ Nucl Med. 2018; 59:459-465.

16. Gaertner FC, Halabi K, Ahmadzadehfar H, et al. Uptake of PSMA-ligands in normal tissues is dependent on tumor load in patients with prostate cancer. Oncotarget. 2017;8:55094-55103.

17. Filss C, Heinzel A, Miiller B, Vogg ATJ, Langen K-J, Mottaghy FM. Relevant tumor sink effect in prostate cancer patients receiving ${ }^{177} \mathrm{Lu}$-PSMA-617 radioligand therapy. Nuklearmedizin. 2018;57:19-25.

18. Sartor O, de Bono JS. Metastatic prostate cancer. N Engl J Med. 2018;378:645657.

19. Fendler WP, Stuparu AD, Evans-Axelsson S, et al. Establishing ${ }^{177}$ Lu-PSMA617 radioligand therapy in a syngeneic model of murine prostate cancer. $J$ Nucl Med. 2017;58:1786-1792. 